QCD Evolution Workshop 2014

International Journal of Modern Physics: Conference Series

Vol. 37 (2015) 1560067 (8 pages)

(C) The Author

DOI: $10.1142 / \mathrm{S} 2010194515600678$

\title{
Target Single-Spin Asymmetry in Inclusive Hadron Production
}

\author{
Xiaodong Jiang \\ Los Alamos National Laboratory, \\ Los Alamos, New Mexico 87545, USA \\ Published 25 February 2015
}

\begin{abstract}
We present the first measurement of target single-spin asymmetries $\left(A_{N}\right)$ in the inclusive hadron production reaction, $\mathrm{e}+{ }^{3} \mathrm{He}^{\uparrow} \rightarrow \mathrm{h}+\mathrm{X}$, using a transversely polarized ${ }^{3} \mathrm{He}$ target at an electron-nucleon center-of-mass energy $\sqrt{s}=3.45 \mathrm{GeV}$. The experiment was conducted at Jefferson Lab in Hall A using a 5.9-GeV electron beam. Several types of hadrons $\left(\pi^{ \pm}, K^{ \pm}\right.$and proton) were detected with an average momentum $\left\langle P_{h}\right\rangle=2.35$ $\mathrm{GeV} / \mathrm{c}$, and an average transverse momentum $\left\langle p_{T}\right\rangle=0.64 \mathrm{GeV} / \mathrm{c}$. The observed asymmetry strongly depends on the type of hadron. A positive asymmetry is observed for $\pi^{+}$and $K^{+}$. A negative asymmetry is observed for $\pi^{-}$. The $\pi^{+}$and $\pi^{-}$asymmetries measured for the ${ }^{3} \mathrm{He}$ target and extracted the "effective- neutron" SSA. Amazingly, we found that the ratio of our observed SSA between $\pi^{+}$and $\pi^{-}$productions closely resemble the ratio of up- to down-quark's contributions to neutron's anomalous magnetic moment.
\end{abstract}

Keywords: Single-spin asymmetry; hadron production; quark Sivers distribution; valence quark.

PACS numbers: 25.30.Fj, 14.20.Dh, 24.85.+p, 25.30.Rw

\section{Introduction}

Several decades of experiments on deep inelastic scattering (DIS) have provided us with detailed information on how partons share the momentum of a fast-moving nucleon. However, we do not have a clear answer to the question of how partons share the nucleon's 1/2- spin. The puzzle of nucleon's "missing-spin" has been hunting theorists and experimentalists alike since the mid-1980's.

In addition to the "missing spin" puzzle, there's another long-standing unexplained spin phenomenon, namely, the observed large transverse single-spin asymmetries (SSA). Surprisingly, large transverse single spin asymmetries (TSSAs), were observed in $p^{\uparrow}+p \rightarrow \pi+X$ reactions first at ZGS in $1970 \mathrm{~s},{ }^{1}$ then in $80 \mathrm{~s}-90 \mathrm{~s}$ at $\mathrm{BNL}^{2}$

This is an Open Access article published by World Scientific Publishing Company. It is distributed under the terms of the Creative Commons Attribution 3.0 (CC-BY) License. Further distribution of this work is permitted, provided the original work is properly cited. 
and Fermilab ${ }^{3}$ over a broad range of $\sqrt{s}$, and reconfirmed at RHIC up to $\sqrt{s}=510$ GeV. Collinear perturbative QCD at leading-twist, ${ }^{4}$ which predicted SSA's are of the order of $\alpha_{s}\left(m_{q} / \sqrt{s}\right)$ (where $m_{q}$ is the quark mass) and thus very small, simply could not explain such a large SSA. Two-types of theoretical frameworks have been developed over the past two decades in efforts to resolve the large SSA puzzle: the transverse momentum dependent (TMD) factorization framework and the collinear twist-3 factorization framework.

The field of transverse spin physics has now become the most active area in high energy nuclear physics, generating tremendous excitements on both theoretical and experimental fronts. Over the last decade, new single-spin asymmetry data has become available in inclusive hadron, inclusive jet and di-hadron correlation from high energy $p+p$ collisions at RHIC, and from SIDIS experiments at CERN, DESY and JLab. In this contribution, we briefly discuss the existing single-spin asymmetry data in $p+p$ and in SIDIS reactions, and their common feature of flavor-dependent left-right biases. We also present our recent results on single-spin asymmetry measurement of electron beam induced hadron production off an "effectively polarized neutron" target $\left({ }^{3} \mathrm{He}\right)$, and point out again to the common feature of flavor-dependent left-right biases. Amazingly, we found that the ratio of our observed SSA between $\pi^{+}$and $\pi^{-}$productions closely resemble the ratio of up- to down-quark's contributions to nucleon's anomalous magnetic moment. More details of our measurement can be found in the recent publications of Jefferson Lab Hall A Collaboration. ${ }^{5}$

\section{Left-right Biases Observed in $p^{\uparrow}+p$ Collisions and in SIDIS}

E704 observed SSA is shown in Fig. 1 on the left, and E704's coordinate system definition is illustrated on the right. The asymmetry is defined as: ${ }^{3}$

$$
A_{N}=-\frac{1}{P_{B} \cos (\phi)} \frac{N_{\uparrow}(\phi)-N_{\downarrow}(\phi)}{N_{\uparrow}(\phi)+N_{\downarrow}(\phi)}
$$

TSSA observed by E704 is a nucleon spin dependent left-right bias, on out-going hadrons from the hard collision. The observation indicated that in the center-ofmass frame of the proton-proton collision, viewed along the direction of the polarized proton's momentum, $\pi^{+}$favors the left side of the proton spin vector, whereas $\pi^{-}$ favors the right side of the proton spin vector.

If one takes a careful look at the sign of SIDIS $\pi^{+}$Sivers asymmetry on polarized proton, as reported by HERMS and COMPASS experiments, one observes a similar left-tight bias: in the center-of-mass frame of the proton-lepton collision, viewed along the direction of the polarized proton's momentum, $\pi^{+}$produce in SIDIS reaction favors the left side of the proton spin vector. 

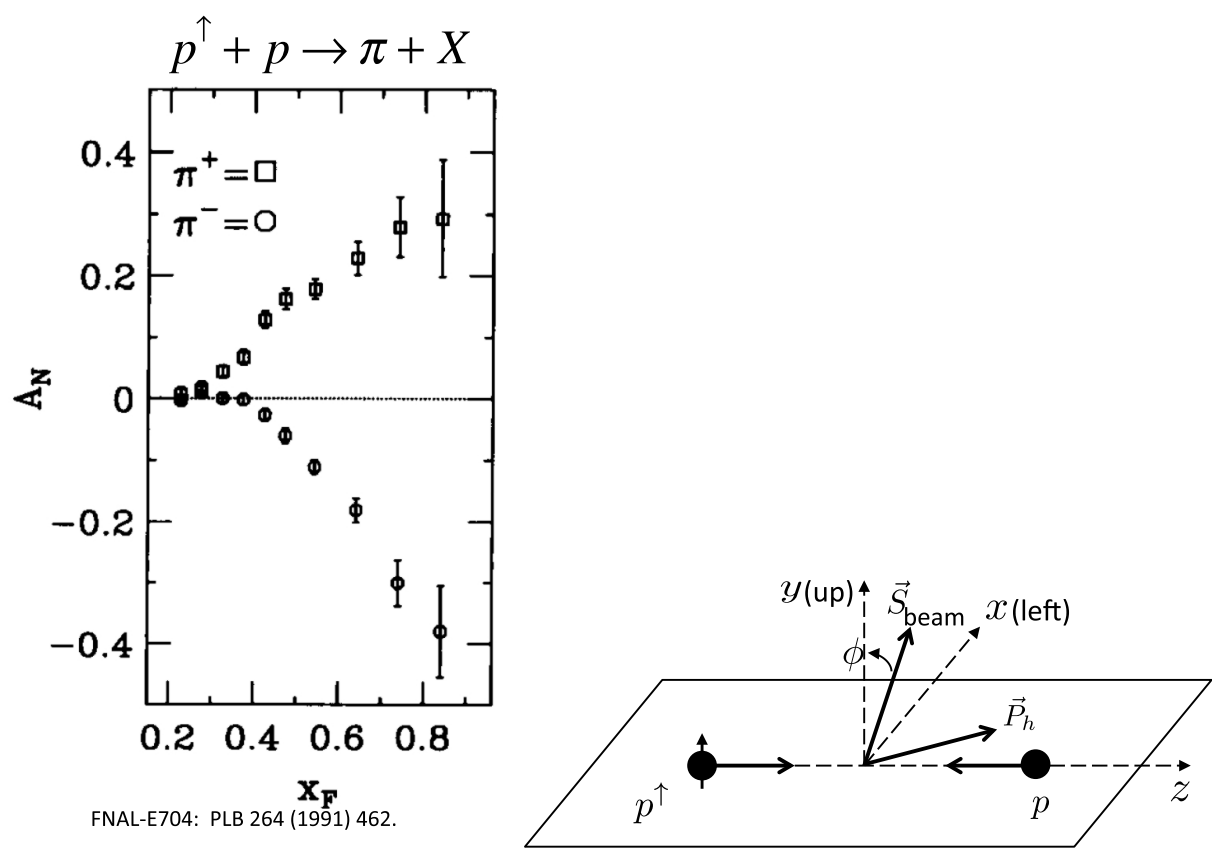

Fig. 1. (left) E704 observed SSA, and (right) the definition of E704's coordinate system.

\section{Left-right Biases Observed in $l+N^{\uparrow} \rightarrow+X$ Reactions}

\subsection{Jefferson lab hall A transversity experiment (E06-010)}

The measurement reported here was carried out from mid October 2008 to early February 2009 at Jefferson Lab Hall A, as part of the "Neutron Transversity" experiment (E06-010). Target single-spin asymmetries $\left(\mathrm{A}_{N}\right)$ in inclusive hadron $\left(\pi^{ \pm}, \mathrm{K}^{ \pm}\right.$, and proton) production were measured at a fixed-target e+ $\mathrm{N}$ center-of-mass energy $\sqrt{s}=3.45 \mathrm{GeV}$, using an unpolarized electron beam (sum over electron beam's helicity) and a transversely polarized ${ }^{3} \mathrm{He}$ target as an effective polarized neutron target. ${ }^{5}$

The kinematical variables for this process are: $x_{F}=2 p^{C M} / \sqrt{s}$, where $p^{C M}$ is the momentum of the outgoing hadron along the polarized nucleon's momentum direction in the e+N center-of-mass frame, and $p_{T}=\sqrt{p_{x}^{2}+p_{y}^{2}}$, the transverse momentum of the outgoing hadron.

\subsection{The coordinate system and the definition of asymmetry}

The kinematical configuration in the laboratory coordinate system is shown in Fig. 2.

The target spin "up" $(\uparrow)$ was defined to be along the $+\hat{y}$ direction, parallel to the vector $\vec{l} \times \overrightarrow{P_{h}}\left(\phi_{S}=90^{\circ}\right)$, where $\vec{l}$ and $\overrightarrow{P_{h}}$ are the momentum vectors of the incoming beam and outgoing hadron, respectively. 


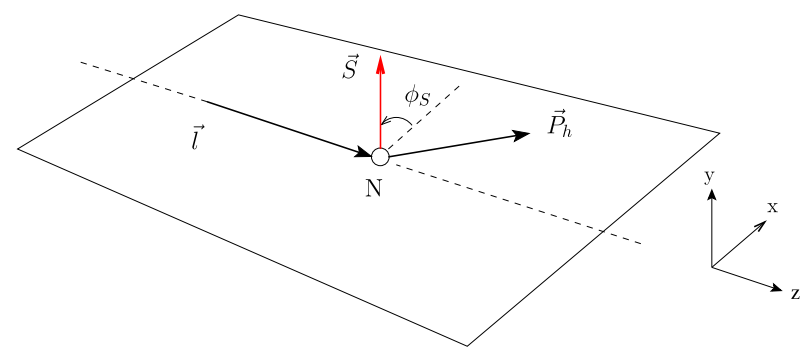

Fig. 2. Kinematical configuration in the laboratory coordinate system for the $l N^{\uparrow} \rightarrow h X$ process. $\vec{P}_{h}$ represents the momentum direction of the produced hadron, and $\vec{S}$ is the spin vector of the nucleon. The polarized nucleon's momentum is along the $-\mathrm{z}$ direction in the e+N center-of-mass frame

The target SSA is defined as ${ }^{6}$

$$
A_{U T}\left(x_{F}, p_{T}\right)=\frac{1}{P} \frac{d \sigma^{\uparrow}-d \sigma^{\downarrow}}{d \sigma^{\uparrow}+d \sigma^{\downarrow}} \sin \phi_{S}=A_{N} \sin \phi_{S}
$$

where $d \sigma^{\uparrow(\downarrow)}$ is the differential cross-section in the target "up" ("down") state, and $P$ is the target polarization. The spin-dependent part of the cross-section is proportional to the term $\vec{S} \cdot\left(\vec{l} \times \overrightarrow{P_{h}}\right)$, which gives rise to a $\sin \left(\phi_{S}\right)$ modulation in the definition of the asymmetry. This term makes $\mathrm{A}_{N}$ parity-conserving, but T-odd under "naïve" time reversal, in which the initial and final states do not interchange. Note that the sign of $A_{N}$ in the laboratory coordinate system of this experiment (Eq. 1) differ by a factor -1 from the definition in the phenomenological study of this process in Anselmino et al. [6], where the authors used the center-of-mass coordinate system with the lepton moving in the $-\hat{z}$ direction.

\subsection{Results}

The final ${ }^{3} \mathrm{He}$ asymmetry results are shown for different hadron species in Fig. 3, the data were integrated in $p_{T}$ and $x_{F}$. The error bars represents the statistical uncertainty. The systematic uncertainties are shown as a solid band.

The measured $\mathrm{A}_{N}$ for $\pi^{+}(\sim 2 \%)$ and $\mathrm{K}^{+}(\sim 6 \%)$ are positive, and opposite in sign to that of $\pi^{-}(\sim 1 \%)$. In addition, the magnitudes of these asymmetries follow $\left|A^{\pi^{-}}\right|<\left|A^{\pi^{+}}\right|<\left|A^{K^{+}}\right|$. The measured $\mathrm{A}_{N}$ for $\mathrm{K}^{-}$and protons was found to be small and consistent with zero. We note that the majority of the detected protons originate through a knock-out reaction from the ${ }^{3} \mathrm{He}$ nucleus, whereas mesons are produced either through fragmentation process or in a photoproduction reaction.

The SSAs for charged pions as a function of $p_{T}$ for a ${ }^{3} \mathrm{He}$ target are shown in Fig. 4. The asymmetry grows as a function of $p_{T}$ and plateaus around $p_{T} \simeq 0.63 \mathrm{GeV} / c$.

We extracted $A_{N}$ on "neutron" from the measured ${ }^{3} \mathrm{He}$ asymmetry using the effective polarization approach, previously used for both inclusive and semi-inclusive 


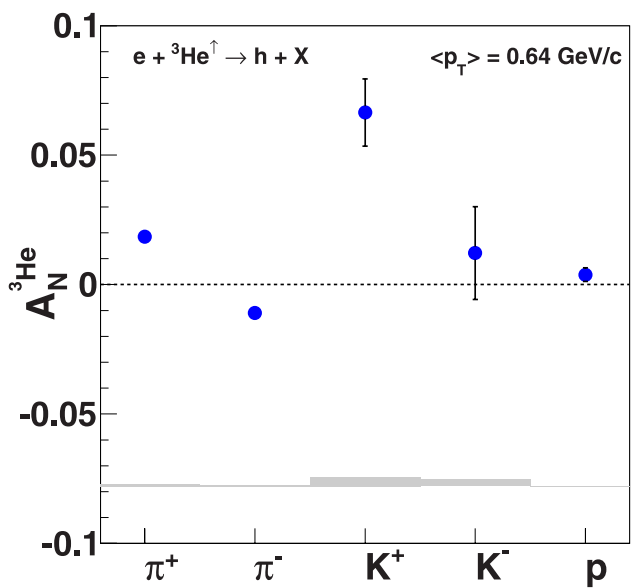

Fig. 3. Inclusive SSA results on a ${ }^{3} \mathrm{He}$ target for $\pi^{ \pm}, \mathrm{K}^{ \pm}$and protons in the vertical target spin configuration $\left(\phi_{S}= \pm 90^{\circ}\right)$. The error bars on the points represents the statistical uncertainty. The grey band shows the magnitude of the overall systematic uncertainty for each hadron channel.

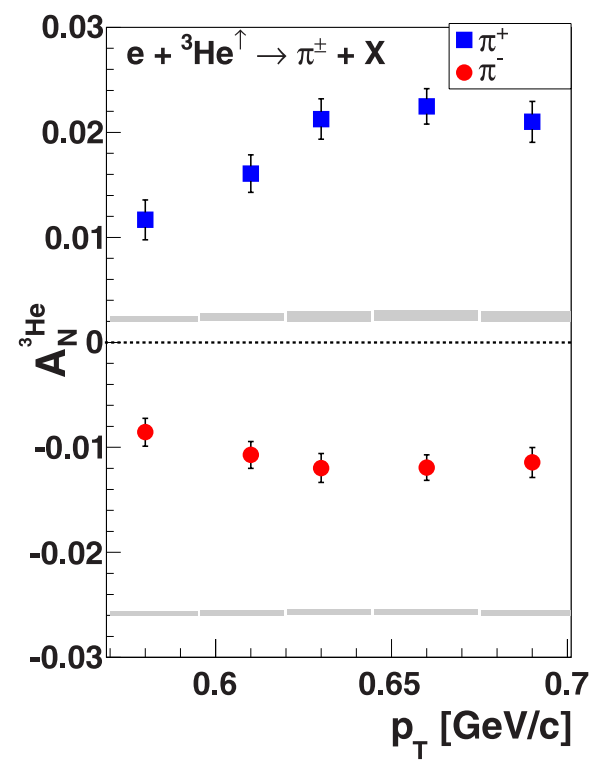

Fig. 4. $A_{N}$ results on a ${ }^{3} \mathrm{He}$ target for the $\pi^{ \pm}$channel as a function of $p_{T}$. The solid band on the bottom of each panel shows the magnitude of the systematic uncertainty for each momentum bin.

DIS processes. Using this method, $A_{N}$ for the neutron can be obtained from ${ }^{3} \mathrm{He}$ results using the relation,

$$
\mathrm{A}_{\mathrm{N}}^{3} \mathrm{He}=\mathrm{P}_{n}\left(1-f_{p}\right) \mathrm{A}_{\mathrm{N}}^{n}+\mathrm{P}_{p} f_{p} \mathrm{~A}_{\mathrm{N}}^{p}
$$




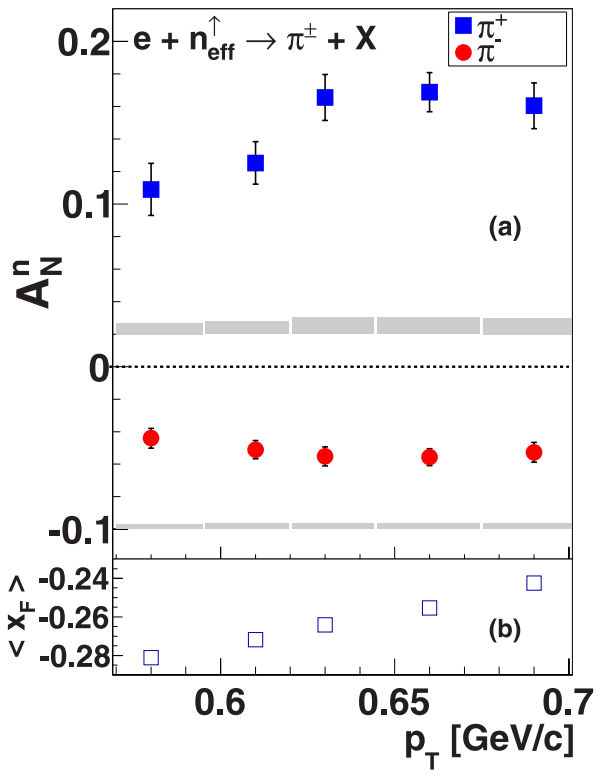

Fig. 5. (a) $\mathrm{A}_{N}$ results on a neutron target extracted from the measured ${ }^{3} \mathrm{He}$ asymmetries. The solid band on the bottom of each panel shows the magnitude of the systematic uncertainty for each momentum bin. The lower plot (b) is the $x_{F}$ and $p_{T}$ correlation in this measurement.

where $\mathrm{A}_{\mathrm{N}}^{3} \mathrm{He}$ is the measured ${ }^{3} \mathrm{He}$ asymmetry. $\mathrm{P}_{n}=0.86_{-0.02}^{+0.036}$ and $\mathrm{P}_{p}=-0.028_{-0.004}^{+0.009}$ are the effective polarization of the neutron and proton, respectively. Hence, the contribution of proton polarization $(\simeq 2.8 \%)$ to $\mathrm{A}_{\mathrm{N}}^{3} \mathrm{He}$ is relatively small. The factor, $f_{p}=2 \sigma_{p} / \sigma_{3}{ }_{H e}$, in ${ }^{3} \mathrm{He}$ was measured directly in this experiment using the yields obtained from unpolarized hydrogen and ${ }^{3} \mathrm{He}$ targets. The average proton dilution $\left(1-f_{p}\right)$ for $\pi^{+}$was $0.156 \pm 0.007$ and for $\pi^{-}$it was $0.268 \pm 0.005$.

The final results for $A_{N}^{n}$ for charged pions on an effective neutron target are shown in Fig. 5. The extracted $A_{N}^{n}$ is below $20 \%$ for both $\pi^{+}$and $\pi^{-}$, with the asymmtry amplitude for $\pi^{+}$being larger than those for $\pi^{-}$. The $A_{N}^{n}$ for both $\pi^{+}$ and $\pi^{-}$increase up to $p_{T} \simeq 0.63 \mathrm{GeV} / \mathrm{c}$, before it plateaus.

\subsection{Discussions}

Currently there are no theoretical estimates for $\mathrm{A}_{N}$ at $\sqrt{s}=3.45 \mathrm{GeV}$ and $\left\langle p_{T}>\sim\right.$ $0.64 \mathrm{GeV} / \mathrm{c}$ for a neutron target. The existing predictions were done for a proton target at $p_{T}=1.5 \mathrm{GeV} / \mathrm{c}$ and $\sqrt{s} \simeq 4.9 \mathrm{GeV} .{ }^{6}$ However, the sign of $\mathrm{A}_{N}$ for $\pi^{ \pm}$in our experiment is consistent with the existing predictions dominated by the Sivers effect, assuming isospin symmetry $\mathrm{p} \leftrightarrow \mathrm{n}$ and $\pi^{+} \leftrightarrow \pi^{-}$.

Our results show that in the center-of-mass frame of the polarized neutronelectron collision, viewed along the direction of the neutron's momentum, $\pi^{+}$favors the right side of the spin vector, whereas $\pi^{-}$favors the left side of the spin vector. 
Table 1. The extracted neutron $\mathrm{A}_{N}^{n}$ results for $\pi^{+}$and $\pi^{-}$along with their ratio $\mathrm{R}_{\pi^{+} / \pi^{-}}$in five different $\left\langle p_{T}\right\rangle$ bins.

\begin{tabular}{cccc}
\hline $\begin{array}{c}<p_{T}> \\
(\mathrm{GeV} / \mathrm{c})\end{array}$ & $A_{N}^{n}\left(\pi^{+}\right) \pm$Stat. \pm Sys. & $A_{N}^{n}\left(\pi^{-}\right) \pm$Stat. \pm Sys. & $\mathrm{R}_{\pi^{+} / \pi^{-}}$ \\
\hline 0.58 & $0.109 \pm 0.016 \pm 0.007$ & $-0.044 \pm 0.006 \pm 0.003$ & $-2.5 \pm 0.5$ \\
0.61 & $0.125 \pm 0.013 \pm 0.008$ & $-0.051 \pm 0.005 \pm 0.003$ & $-2.5 \pm 0.4$ \\
0.63 & $0.166 \pm 0.014 \pm 0.010$ & $-0.055 \pm 0.006 \pm 0.004$ & $-3.0 \pm 0.5$ \\
0.66 & $0.169 \pm 0.012 \pm 0.010$ & $-0.056 \pm 0.005 \pm 0.004$ & $-3.0 \pm 0.4$ \\
0.69 & $0.160 \pm 0.014 \pm 0.010$ & $-0.053 \pm 0.006 \pm 0.003$ & $-3.0 \pm 0.5$ \\
\hline
\end{tabular}

\begin{abstract}
Assuming isospin symmetry, this behavior is the same as that observed in $p p^{\uparrow} \rightarrow h X$ for the E704 and BRAHMS ${ }^{7}$ experiments. In addition, this behavior is also the same as the Sivers asymmetry for $\pi^{+}$observed in SIDIS. The $\mathrm{A}_{N}^{n}$ for $\pi^{+}$is about $\sim 15 \%$ at $\left\langle p_{T}\right\rangle=0.64 \mathrm{GeV} / \mathrm{c}$, which is larger than that for HERMES proton data for $\pi^{+}$ $\left(\sim 5 \%\right.$ at $\left\langle p_{T}>=0.68 \mathrm{GeV} / \mathrm{c}\right){ }^{8}$ Similarly, we observed large $\mathrm{A}_{N}^{n}$ for $\pi^{-}(\sim 5 \%)$ compared to that for HERMES proton data $(<1 \%)[8]$. Furthermore, we observed a large and positive amplitude for the $\mathrm{K}^{+}$asymmetry compared to $\mathrm{K}^{-}$asymmetry on ${ }^{3} \mathrm{He}$, a similar feature observed in $l p^{\uparrow} \rightarrow h X$ reaction on proton target, ${ }^{8}$ and also the Sivers amplitude for kaons in the SIDIS reaction at HERMES. ${ }^{9}$

If these non-zero asymmetries survive at high energy kinematics then they can be used as a practical way to monitor transverse target polarization in a fixed target experiment, or to monitor local transverse polarization of a polarized ${ }^{3} \mathrm{He}$ beam at a future Electron-Ion-Collider.
\end{abstract}

\title{
3.5. SSA and nucleon's anomalous magnetic moment
}

These large TSSAs in $l+N^{\uparrow} \rightarrow \pi^{ \pm}+X$ are rather surprising. The strong flavor dependency and their consistencies in left-right biases with $p+p$ and SIDIS data suggest that these asymmetries have a common origin, for example, from effects of valence quarks' transverse motion.

In addition, the relative size between $\pi^{+}$and $\pi^{-}$asymmetries seems to be wellrelated to each other. We list the numerical values of these asymmetries, and their ratios $\mathrm{R}_{\pi^{+} / \pi^{-}}$in Table 1 . Within our limited $p_{T}$ coverage, the values are rather stable at $\left|R_{\pi^{+} / \pi^{-}}\right| \approx 2.5 \sim 3.0$.

Are the observed SSA related to nucleon's anomalous magnetic moments, as proposed by $\mathrm{Lu}$ and Schmidt [10]? One can write $u$ and $d$ quarks contributions to the anomalous moments of proton and neutron $\left(\kappa_{p}=1.79, \kappa_{n}=-1.91\right)$. Isospin symmetry implies

$$
\begin{aligned}
\kappa_{d / n} & =\kappa_{u / p}, \\
\kappa_{u / n} & =\kappa_{d / p} .
\end{aligned}
$$


In the valence quark approximation we have:

$$
\begin{aligned}
& \kappa_{p}=(2)(2 / 3) \kappa_{u / p}+(-1 / 3) \kappa_{d / p}, \\
& \kappa_{n}=(2)(-1 / 3) \kappa_{u / p}+(2 / 3) \kappa_{d / p} .
\end{aligned}
$$

Thus one has $\kappa_{u / p}=0.835, \kappa_{d / p}=-2.03$. Simply plug these values into the last equation, we obtain the ratio of second-term/first-term=-2.43, i.e. the ratio of upand down-quark's contribution to neutron's anomalous magnetic moments, which is reasonably close to the value of $\mathrm{R}_{\pi^{+} / \pi^{-}}$observed in our measurement.

\section{Conclusions}

We presented our results on single-spin asymmetry measurement of electron beam induced hadron production off an "effectively polarized neutron" target $\left({ }^{3} \mathrm{He}\right)$, and point out the common feature of flavor-dependent left-right biases between $p^{\uparrow}+p$, SIDIS and in $l+N^{\uparrow}$. We found that the ratio of our observed SSA between $\pi^{+}$and $\pi^{-}$productions closely resemble the ratio of up- to down-quark's contributions to neutron's anomalous magnetic moment.

\section{References}

1. R. D. Klem, et al., Phys. Rev. Lett. 36, 929 (1976).

2. C. E. Allgower et al., Phys. Rev. D 65, 092008 (2002).

3. D. Adams et al., Phys. Lett. B, 265, 462 (1991).

4. G. L. Kane, J. Pumplin, and W. Repko, Phys. Rev. Lett., 41, 1689 (1978).

5. K. Allada et al., (Jefferson Lab Hall A Collaboration) Phys. Rev. C. 89, 042201(R) (2014).

6. M. Anselmino et al., Phys. Rev. D. 81, 034007 (2010).

7. I. Arsene et al., Phys. Rev. Lett. 101, 042001 (2008).

8. A. Airapetian et al., Phys. Lett. B 728, 183 (2014).

9. A. Airapetian et al., Phys. Rev. Lett. 103, 152002 (2009).

10. Z. Lu and I. Schmidt, Phys. Rev. D 75, 073008 (2007). 